\title{
Interferência do Controle Glicêmico na Transição entre as Fases I e II da Lactogênese em Pacientes com Diabetes Melito Tipo 1
}

\begin{abstract}
RESUMO
A lactogênese é constituída pelas fases designadas como I e II. A transição entre essas fases é caracterizada pelo aumento da concentração de lactose no colostro. Este estudo teve como objetivo analisar a interferência do diabetes melito tipo 1 (DM1) na transição entre as fases da lactogênese. A concentração de lactose foi determinada em amostras de colostro de 11 puérperas portadoras de DM1 e de 19 puérperas sem a doença, durante os cinco primeiros dias do puerpério. A determinação da concentração da lactose foi feita pela reação com ácido pícrico. Em ambos os grupos houve aumento progressivo da concentração da lactose em função do tempo; entretanto, o aumento foi significativamente menor no terceiro e no quinto dia no grupo das portadoras de diabetes. A análise da transição de fases da lactogênese revelou que as puérperas portadoras de diabetes melito com controle glicêmico inadequado apresentaram atraso de 18 horas para alcançar a fase II da lactogênese, dificultando o estabelecimento do aleitamento materno. (Arq Bras Endocrinol Metab 2008; 52/3:473-481)
\end{abstract}

Descritores: Diabetes melito; Leite humano; Lactogênese; Lactose

\begin{abstract}
Interference of the Blood Glucose Control in the Transition Between Phases I and II of Lactogenesis in Patients with Type 1 Diabetes Mellitus.

Lactogenesis is constituted by phases I and II. The transition between those phases is characterized by an increase of the lactose concentration in the colostrum. This study aimed to evaluate the interference of type 1 Diabetes mellitus in the transition between phases I and II of the lactogenesis. The lactose concentration was determined in colostrum samples of 11 puerperal women with pre-gestational Diabetes mellitus and 19 without the disease, during the five first days of the puerperium. The lactose concentration was determined by reaction with picric acid. In both groups there were progressive increases in the lactose levels along the time; however, the increase was significantly lower in the $3^{\text {rd }}$ and $5^{\text {th }}$ days for the group of the women with diabetes. The analysis of the transition between the lactogenesis phases revealed that the puerperal diabetic women with inadequate glycemic control presented a time delay of 18 hours to reach phase II, making difficult the establishment of breastfeeding. (Arq Bras Endocrinol Metab 2008; 52/3:473-481)
\end{abstract}

Keywords: Diabetes mellitus; Human milk; Lactogenesis; Lactose artigo original

\author{
Angela M. de Morais Oliveira \\ Cleine Chagas da Cunha \\ NiLson PENha-SILVA \\ VÂnia Olivetti Steffen Abdallah \\ Paulo tannus Jorge
}

Banco de Leite Humano do

Hospital das Clínicas da

Universidade Federal de

Uberlândia (UFU), MG, Brasil

(AMMO); Instituto de Genética e

Bioquímica da UFU (CCC, NPS);

Departamento de Pediatria,

Serviço de Neonatologia da Faculdade de Medicina da UFU

(VOSA); Departamento de

Clínica Médica, Serviço de

Endocrinologia da Faculdade de Medicina da UFU (PTJ), MG, Brasil.

Recebido em 28/09/2006

Aceito em 03/08/2007 


\section{INTRODUÇÃO}

LACTOGÊNESE É GERALMENTE dividida em duas fases, lactogênese I e lactogênese II. Em humanos, a fase I ocorre a partir da $20^{\mathrm{a}}$ semana de gravidez (1) e pode ser detectada pela medida do aumento da concentração plasmática de lactose e $\alpha$-lactalbumina (2). Poucos mililitros de um líquido amarelado são secretados desde o último trimestre da gestação e na primeira semana pósparto. $\mathrm{O}$ volume deste líquido, geralmente chamado colostro, varia 10 a $100 \mathrm{ml} /$ dia $(3,4)$.

A fase II da lactogênese é caracterizada pelo início de abundante secreção de leite e está associada a grandes mudanças na composição da secreção mamária que caracteriza a transição de colostro para leite maduro. O segundo estágio da lactogênese, normalmente, ocorre entre 24 e 48 horas após o parto $(1,5,6)$.

A lactose é o carboidrato predominante no leite. É sintetizada pela glândula mamária e constitui cerca de $70 \%$ do conteúdo de carboidratos do leite humano. A concentração de lactose varia de 4 a $5,3 \mathrm{~g} / \mathrm{dl}$, no colostro, ou $7 \mathrm{~g} / \mathrm{dl}$, no leite maduro (3,7-9). A concentração de lactose no leite humano tem sido utilizada como marcador do início da lactogênese II $(10,11)$.

As vantagens da amamentação são muitas e estão documentadas na literatura. Vários trabalhos mostram o efeito protetor do leite humano contra a morbi mortalidade infantil. Sabe-se, por exemplo, que certas infecções, principalmente nos tratos respiratório e digestivo, alergias nutricionais e doenças crônicas, ocorrem menos freqüentemente em crianças que são amamentadas $(5,12,13)$. A amamentação também contribui para a recuperação da mulher no puerpério e na proteção contra o câncer de mama (14).

A capacidade da mulher com diabetes melito tipo 1 (DMl) em engravidar e prolongar a gravidez a termo tem sido aumentada pelo melhoramento do controle metabólico (15). As mulheres portadoras de diabetes melito têm sido encorajadas a amamentar $(16,17)$ por razões que incluem uma possível redução do risco de DMl em seus filhos (18). A pequena duração da amamentação, com a introdução precoce do leite de vaca foi apontada como um fator de risco para o desenvolvimento do DMl em crianças (19). A ausência da amamentação foi associada com o risco de $\mathrm{DMl}$ em uma população com rápido aumento da incidência da doença (20). O aumento da duração da amamentação também foi associado com a redução do risco futuro de diabetes melito do tipo 2 (DM2) na mulher que amamentou (21).
A excelência do leite humano como alimento para a criança recém-nascida não é motivo de dúvidas. A World Health Organization (22) recomenda que o aleitamento materno seja a única fonte de alimento nos primeiros 6 meses de vida da criança e que seja estendido até os 2 anos ou mais. A American Dietetic Association (23) também se posicionou a favor da amamentação, ressaltando os benefícios, tanto para as crianças quanto para as mães que amamentaram.

Este estudo teve como objetivo investigar a interferência do DMl na transição da lactogênese I para a lactogênese II por meio da determinação da concentração de lactose no colostro de mulheres puérperas.

\section{MATERIAL E MÉTODOS}

Este estudo foi aprovado pelo Comitê de Ética em Pesquisa da Universidade Federal de Uberlândia e devidamente autorizado pelas pacientes que assinaram um termo de consentimento livre e esclarecido. $\mathrm{O}$ estudo foi desenvolvido no período de janeiro a dezembro de 2004.

Foram incluídas no estudo todas as puérperas portadoras de diabetes melito (PPDM) do tipo 1 pré-gestacional que realizaram o parto no Serviço Especializado em Gestação de Alto Risco do Hospital de Clínicas da Universidade Federal de Uberlândia (HC-UFU) durante o período mencionado anteriormente. $\mathrm{O}$ diabetes foi diagnosticado com base no Consenso Brasileiro de Diabetes (24) ou nas recomendações da American Diabetes Association (25). O grupo-controle foi composto por puérperas sem diabetes (PSD), com glicemia de jejum < $85 \mathrm{mg} / \mathrm{dl}$ ou glicemia < $140 \mathrm{mg} / \mathrm{dl}$ duas horas após a ingestão de $75 \mathrm{~g}$ de glicose, segundo o Consenso Brasileiro de Diabetes (24). Os grupos foram pareados de acordo com a idade materna, índice de massa corporal (IMC) pré-gestacional, ganho de peso durante a gestação, tipo e número de partos e idade gestacional do recém-nascido. Totalizaram 11 puérperas portadoras de DMl pré-gestacional e 19 puérperas sem diabetes.

O controle glicêmico das pacientes PPDM foi avaliado pela média dos valores das glicemias capilares de jejum e pós-prandiais ( 2 horas após café da manhã, almoço, jantar e lanche noturno), mensuradas em um glicosímetro Roche ${ }^{\circledR}$, modelo ACCU-Chek Active, nos três primeiros dias do puerpério. As médias dos valores da glicemia de jejum $<140 \mathrm{mg} / \mathrm{dl}$ e pós-prandial $<180 \mathrm{mg} / \mathrm{dl}$ foram consideradas como controle metabólico adequado (26). 


\section{Coleta das amostras de colostro e determinação da concentração de lactose}

As amostras de colostro $(3 \mathrm{ml})$ foram coletadas, por expressão manual, diariamente durante os primeiros cinco dias do puerpério, foram acondicionadas em copo de polipropileno e congeladas a $20{ }^{\circ} \mathrm{C}$ negativos para análise posterior.

A concentração de lactose nas amostras de colostro foi determinada usando o método colorimétrico fundamentado na reação com ácido pícrico, como descrito na literatura $(27,28)$. Como este método é de natureza oxirredutimétrica, também determina a concentração de outros açúcares redutores em amostras de colostro. Para avaliar se os níveis de glicose do colostro afetariam de maneira significante os valores determinados para a concentração de lactose, também determinamos a concentração de glicose em 25 amostras de colostro de PPDM e 25 amostras de colostro de PSD, segundo o método da glicose-oxidase, específico para dosagem de glicose (29).

\section{Análises estatísticas}

As análises estatísticas foram realizadas com o uso do software Origin 6.0 (Microcal Inc., Northampton, Massachusetts, EUA). Os dados das PPDM e PSD foram comparados utilizando o teste $t$ de Student (30), $\operatorname{com} \mathrm{p}<0,05$ indicando diferenças estatisticamente significante. As freqüências relativas utilizadas para comparar a idade gestacional do recém-nascido, o local de permanência do recém-nascido e o horário da primeira mamada no seio materno foram analisados com base no teste não-paramétrico de Fisher (31) com o nível de significância estabelecido em $5 \%$, usando a prova bilateral. A dependência da concentração de lactose no colostro em função do tempo após o parto foi ajustada a uma linha de transição sigmoidal, definida pela equação de Boltzmann aplicada para este modelo:

$$
\text { (Lactose) }=\frac{a_{1}-a_{2}}{1+e^{\left(t-t_{0,5}\right) / d t}}+a_{2}
$$

em que $a_{1}$ e $a_{2}$ representam respectivamente o mínimo (lactogênese I) e máximo (lactogênese II) de concentração de lactose, $\mathrm{t}_{0,5}$ é o tempo no ponto intermediário da linha de transição e dt representa a amplitude da transição (Figura 1). A região intermediária linear da sigmóide foi também ajustada pela regressão linear para permitir a determinação de $t_{t}$, tempo total necessário para completar a transição da lactogênese I para a lactogênese II. O valor de $t_{t}$ foi obtido pelo valor na abscissa do ponto de interseção da reta de regressão linear com $a_{2}$.

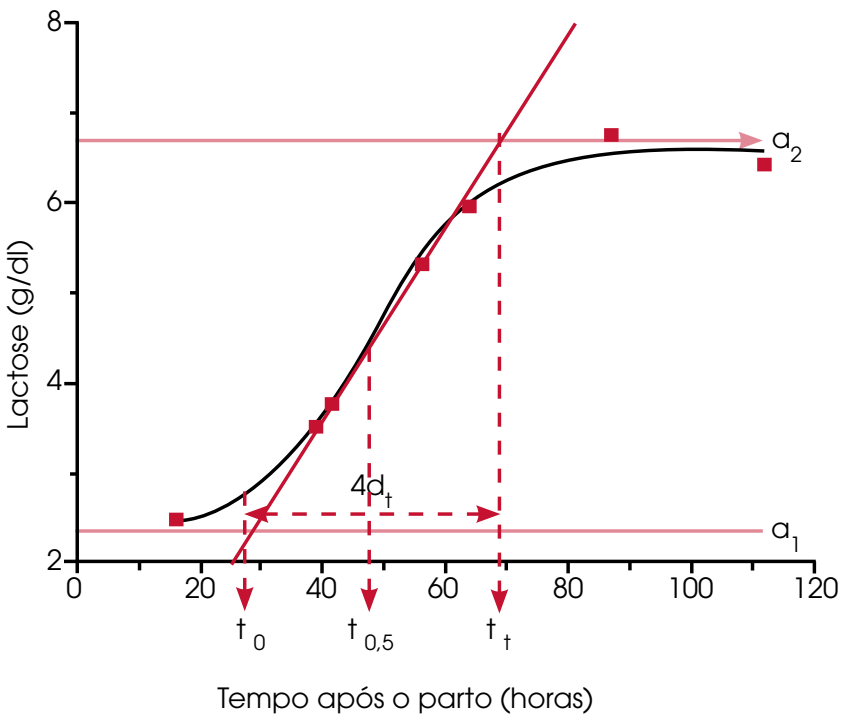

Figura 1. Representação do ajuste sigmoidal da transição entre as fases I e II da lactogênese, com definição dos parâmetros $a_{1}$ (concentração média de lactose na lactogênese 1 ), $a_{2}$ (concentração média de lactose na lactogênese II), $t_{0}$ (tempo inicial da transição), $t_{0,5}$ (tempo de meia transição), $t_{+}$(tempo total necessário para atingir a concentração média de lactose na lactogênese II), e $4 d_{\dagger}(4$ vezes $d_{\dagger}$ é o valor da amplitude da transição).

\section{RESULTADOS}

\section{Características das mulheres puérperas}

As pacientes PPDM tinham idade entre 17 e 29 anos ( édia $=22$ anos) e um tempo de diagnóstico da doença entre 4 e 18 anos (média $=11$ anos). Não houve diferença estatisticamente significante entre os grupos de PPDM e PSD em relação à idade, ao tipo e ao número de partos, ao IMC e ao ganho de peso durante a gestação (Tabela 1). As pacientes dos dois grupos foram submetidas a parto por cesariana.

Todas as PPDM faziam uso de insulina antes da gravidez. Eram primíparas nove $(82 \%)$; cinco $(45,5 \%)$ tinham complicações crônicas do diabetes, como retinopatia, nefropatia ou neuropatia; duas (18\%) apresentavam hipertensão arterial; e uma $(9,1 \%)$ apresentava insuficiência cardíaca congestiva. Apresentaram controle glicêmico adequado cinco PPDM $(45,5 \%)$ e seis PPDM $(54,5 \%)$ apresentaram controle glicêmico inadequado nos primeiros três dias do puerpério (Tabela 2 ). 
Tabela 1. Característica da PPDM, da PSD e de seus filhos recém-nascidos.

\begin{tabular}{|c|c|c|c|}
\hline Características & $\begin{array}{c}\text { PPDM }(n=11) \\
\text { Média } \pm \text { DP } \\
\text { (intervalo) }\end{array}$ & $\begin{array}{c}\text { PSD }(n=19) \\
\text { Média } \pm \text { DP } \\
\text { (intervalo) }\end{array}$ & $p^{a}$ \\
\hline Idade (anos) & $\begin{array}{c}22,0 \pm 4,1 \\
(17-29)\end{array}$ & $\begin{array}{c}25,7 \pm 5,5 \\
(17-38)\end{array}$ & 0,062 \\
\hline IMC pré-gestacional ${ }^{b}$ & $\begin{array}{c}22,4 \pm 3,2 \\
(18-30)\end{array}$ & $\begin{array}{c}21,4 \pm 3,4 \\
(15-29)\end{array}$ & 0,431 \\
\hline Ganho de peso (kg) & $\begin{array}{l}11,7 \pm 4,5 \\
(4,8-18,5)\end{array}$ & $\begin{array}{c}14,0 \pm 4,8 \\
(8-25)\end{array}$ & 0,209 \\
\hline Número de partos & $\begin{array}{c}1,3 \pm 0,6 \\
(1-3)\end{array}$ & $\begin{array}{c}1,9 \pm 0,9 \\
(1-4)\end{array}$ & 0,074 \\
\hline Idade gestacional RN (dias) & $\begin{array}{c}262 \pm 8 \\
(244-273)\end{array}$ & $\begin{array}{c}268 \pm 10 \\
(246-281)\end{array}$ & 0,114 \\
\hline Peso nascimento RN (g) & $\begin{array}{l}3.496 \pm 839 \\
(2.000-4.600)\end{array}$ & $\begin{array}{c}2.772 \pm 542 \\
(1.800-3.780)\end{array}$ & 0,007 \\
\hline
\end{tabular}

a Teste $t$ de Student; ${ }^{\circ}$ IMC = índice de massa corporal; PPDM = puérpera portadora de diabetes melito); PSD = puérpera sem diabetes; RN = recém-nascido.

Tabela 2. Valores das concentrações de glicose e creatinina no sangue das puérperas portadoras de diabetes melito.

\begin{tabular}{|c|c|c|c|}
\hline \multirow{2}{*}{ Paciente } & \multicolumn{2}{|c|}{ Glicose (mg/dl) ${ }^{a}$} & \multirow{2}{*}{ Creatinina $(\mathrm{mg} / \mathrm{dl})$} \\
\hline & Jejum & Pós-prandial & \\
\hline 1 & 176 & 120 & 0,7 \\
\hline 2 & 123 & 130 & 1,2 \\
\hline 3 & 127 & 217 & 0,7 \\
\hline 4 & 102 & 112 & 0,8 \\
\hline 5 & 81 & 202 & 0,6 \\
\hline 6 & 91 & 234 & 0,6 \\
\hline 7 & 54 & 170 & 0,7 \\
\hline 8 & 98 & 194 & 0,6 \\
\hline 9 & 109 & 137 & 0,8 \\
\hline 10 & 170 & 206 & 0,8 \\
\hline 11 & 98 & 124 & 0,6 \\
\hline
\end{tabular}

a Média das glicemias nos primeiros três dias do puerpério; jejum e pós-prandial (após café-da-manhã, almoço, jantar e lanche noturno).

\section{Caracterização dos recém-nascidos}

A idade gestacional média dos filhos recém-nascidos das puérperas portadoras de diabetes (FPPDM) foi de 37 semanas e 3 dias (262 dias). Essa idade não foi estatisticamente diferente $(\mathrm{p}=0,11)$ da média da idade gestacional dos filhos das puérperas sem diabetes (FPSD), que foi de 38 semanas e 2 dias (268 dias). Nenhum recém-nascido apresentou idade gestacional menor que 35 semanas. Os FPPDM apresentaram peso ao nascimento significantemente maior $(\mathrm{p}<0,01)$ do que os FPSD (Tabela 1).

A maioria dos FPPDM (91,0\%) permaneceu na unidade neonatal nas primeiras horas após o parto, em decorrência de hipoglicemia neonatal $(100 \%)$, icterícia (73\%) e persistência do canal arterial $(5,3 \%)$. Um número significativamente menor $(\mathrm{p}=0,013)$ dos FPSD $(47,4 \%)$ permaneceu na unidade neonatal, em decorrência de icterícia $(78 \%)$, hipoglicemia neonatal $(55,5 \%)$, 
doença infecciosa $(5,3 \%)$ e taquipnéia transitória do recém-nascido $(5,3 \%)$.

Durante os primeiros cinco dias após o parto, todos os recém-nascidos foram amamentados por suas mães. Os FPPDM também receberam uma dieta complementar. No grupo dos FPSD, $52,6 \%(\mathrm{n}=10)$ foram alimentados exclusivamente ao seio materno e $47,4 \%(\mathrm{n}=9)$ receberam também uma dieta complementar. Esta dieta complementar era leite humano pasteurizado, fornecido pelo Banco de Leite Humano do HC-UFU, ou uma fórmula infantil padrão. Apesar da separação precoce de alguns bebês de suas mães, 66,6\% $(\mathrm{n}=7)$ dos FPPDM e $73,7 \%(\mathrm{n}=14)$ dos FPSD sugaram o seio materno antes de completar 24 horas de vida $(\mathrm{p}=0,285)$.

\section{Concentração de lactose na amostras de colostro}

Não houve diferença estatisticamente significativa no horário de coleta das amostras de colostro, entre os grupos de paciente PPDM e PSD $(\mathrm{p}>0,05)$. As cinco coletas de colostro foram realizadas às $23 \pm 4,6$; $44 \pm 4,6 ; 68 \pm 7 ; 93 \pm 7,5$ e $118 \pm 4$ horas após o parto, no grupo das PPDM, e às $21 \pm 5 ; 45 \pm 5,3 ; 68$ $\pm 5,5 ; 93 \pm 4,9$ e $117 \pm 4$ horas após o parto, no grupo das PSD.

Os valores da concentração de lactose estão apresentados na Tabela 3. Ambos os grupos apresentaram aumento na concentração da lactose do primeiro ao quinto dia do puerpério. No grupo das PPDM foi observada menor concentração de lactose no colostro do que no grupo-controle, no terceiro $(\mathrm{p}<0,01)$ e no quinto dia $(\mathrm{p}<0,01)$ do puerpério.

A concentração de lactose no colostro das PSD não foi significativamente diferente $(p>0,05)$ se os recém-nascidos permaneceram no sistema de alojamento conjunto $(\mathrm{n}=10)$ ou se os recém-nascidos foram internados na unidade neonatal $(\mathrm{n}=9)$, como pode ser visto na Tabela 4.

Tabela 3. Concentração de lactose (g/dl) no colostro de PPDM e de PSD, nos cinco primeiros dias do puerpério.

\begin{tabular}{lccc}
\hline Dia & $\begin{array}{c}\text { PPDM }(n=11) \\
\text { Média } \pm \text { DP }\end{array}$ & PSD $(n=19)$ \\
Média \pm DP & $p^{a}$ & 0,447 \\
$1^{\circ}$ & $1,48 \pm 1,44$ & $1,90 \pm 1,46$ & 0,300 \\
\hline $2^{\circ}$ & $3,09 \pm 2,19$ & $3,96 \pm 2,12$ & 0,008 \\
\hline $3^{\circ}$ & $5,15 \pm 1,91$ & $6,72 \pm 1,13$ & 0,162 \\
\hline $4^{\circ}$ & $6,27 \pm 0,87$ & $6,81 \pm 1,05$ & 0,008 \\
\hline $5^{\circ}$ & $5,98 \pm 0,88$ & $6,91 \pm 0,86$ & \\
\hline
\end{tabular}

a Teste $t$ de Student; PPDM = puérpera portadora de diabetes melito; PSD = puérpera sem diabetes.

Tabela 4. Concentração de lactose ( $\mathrm{g} / \mathrm{dl})$ no colostro de PSD nos primeiros cinco dias do puerpério, conforme o local de permanência do recém-nascido.

\begin{tabular}{|c|c|c|c|}
\hline Dia & $\begin{array}{l}\text { FPSD no alojamento conjunto }(n=10) \\
\text { Média } \pm \text { DP }\end{array}$ & $\begin{array}{l}\text { FPSD na unidade neonatal }(n=9) \\
\text { Média } \pm \text { DP }\end{array}$ & $p^{a}$ \\
\hline $1^{\circ}$ & $1,68 \pm 1,31$ & $2,16 \pm 1,66$ & 0,492 \\
\hline $2^{\circ}$ & $4,05 \pm 2,68$ & $3,86 \pm 1,42$ & 0,851 \\
\hline $3^{\circ}$ & $6,54 \pm 0,56$ & $6,92 \pm 1,56$ & 0,487 \\
\hline $4^{\circ}$ & $6,98 \pm 0,68$ & $6,62 \pm 1,37$ & 0,470 \\
\hline $5^{\circ}$ & $7,03 \pm 0,57$ & $6,79 \pm 1,12$ & 0,562 \\
\hline
\end{tabular}




\section{Caracterização da transição entre as fases I e II da lactogênese}

Uma típica dependência da concentração de lactose no colostro em função do tempo está apresentada com seu ajuste a uma linha de regressão sigmoidal na Figura 1. Invariavelmente, os dados apresentaram ajuste sigmoidal estatisticamente significante $(\mathrm{p}<0,01)$. Os ajustes simultâneos das linhas de regressão sigmoidal para todos os dados em cada grupo estão apresentados na Figura 2, dando exatamente uma idéia sobre a diferença entre os grupos. Os valores obtidos com base na análise de cada paciente, individualmente, estão apresentados na Tabela 5. Não houve diferença significante nos ní-

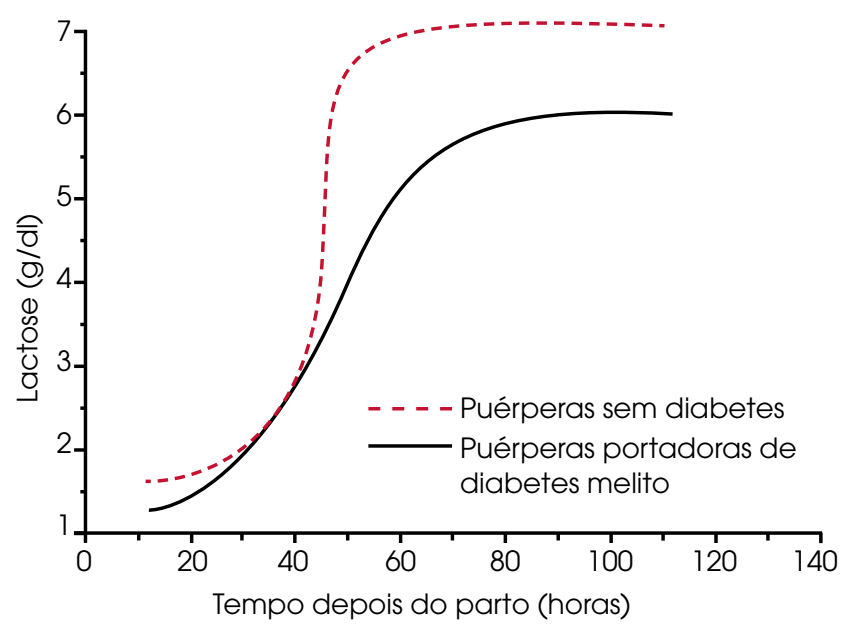

Figura 2. Ajuste simultâneo para a dependência da concentração de lactose no colostro de puérperas sem diabetes (PSD) e puérperas portadoras de diabetes melito (PPDM) com o tempo após o parto. veis de lactose da lactogênese I $\left(a_{1}\right)$, lactogênese II $\left(a_{2}\right)$ e no ponto médio $\left(t_{0,5}\right)$ da transição entre os grupos. Entretanto, o tempo total $\left(\mathrm{t}_{\mathrm{t}}\right)$ para completar a transição entre as lactogêneses I e II foi estatisticamente significantemente maior no grupo das PPDM do que no grupo das PSD $(\mathrm{p}=0,02)$.

As pacientes do grupo das PPDM com controle metabólico adequado apresentaram um valor médio de $\mathrm{t}_{\mathrm{t}}(63,7 \pm 13,3 \mathrm{~h})$ que não foi significativamente diferente em relação ao valor médio de $t_{t}(71,0 \pm 28,0 \mathrm{~h})$ das pacientes do grupo das PPDM com controle metabólico inadequado. Entretanto, os valores de $t_{t}$ foram significativamente diferentes entre as pacientes do grupo das PPDM com controle metabólico inadequado e os valores de $t_{t}$ entre as pacientes do grupo das PSD $(53,0 \pm 11,5 \mathrm{~h})$, como pode ser visto na Tabela 6 .

As concentrações de glicose encontradas no colostro das pacientes de ambos os grupos foram muito pequenas em relação ao nível dos açúcares redutores totais. A maior proporção de glicose encontrada no colostro das pacientes PPDM e de PSD foi, respectivamente, $0,27 \%$ e $0,28 \%$ do valor total de açúcar redutor determinado pelo método do ácido pícrico.

\section{DISCUSSÃo}

Nos primeiros cinco dias do puerpério, os níveis de lactose no colostro das pacientes PPDM e PSD aumentaram seguindo um padrão típico de transição entre dois estados ou fases (Figura 1), em que a concentração de lactose mudou bruscamente dos níveis da lactogênese I

Tabela 5. Valores dos parâmetros que caracterizam a transição entre as fases I e II da lactogênese, obtidos da linha de dependência sigmoidal da concentração de lactose no colostro com o tempo em horas após o parto.

\begin{tabular}{|c|c|c|c|}
\hline Parâmetros & $\begin{array}{c}\text { PPDM }(n=11) \\
\text { Média } \pm \text { DP }\end{array}$ & $\begin{array}{l}\text { PSD }(n=19) \\
\text { Média } \pm \text { DP }\end{array}$ & $p^{a}$ \\
\hline$a_{1}$ & $1,2 \pm 1,3$ & $1,5 \pm 1,7$ & 0,972 \\
\hline$a_{2}$ & $6,23 \pm 0,8$ & $6,9 \pm 0,9$ & 0,060 \\
\hline$t_{0}$ & $39,2 \pm 16,9$ & $33,7 \pm 19,1$ & 0,438 \\
\hline$t_{0,5}$ & $53,0 \pm 16,2$ & $41,7 \pm 15,6$ & 0,112 \\
\hline$d_{t}$ & $6,5 \pm 6,3$ & $4,1 \pm 5,6$ & 0,302 \\
\hline$t_{t}$ & $68,0 \pm 21,9$ & $53,0 \pm 11,5$ & 0,022 \\
\hline
\end{tabular}


Tabela 6. Comparação dos parâmetros que caracterizam a transição entre as fases I e ll da lactogênese entre as pacientes PSD e PPDM conforme o controle glicêmico no puerpério.

\begin{tabular}{|c|c|c|c|}
\hline Parâmetros & $\begin{array}{l}\text { PSD }(n=19) \\
\text { Média } \pm \text { DP }\end{array}$ & $\begin{array}{l}\text { PPDM com controle adequado } \\
(n=5) \text { média } \pm \text { DP }\end{array}$ & $\begin{array}{l}\text { PPDM com controle inadequado } \\
\qquad(n=6) \text { média } \pm \text { DP }\end{array}$ \\
\hline$a_{1}$ & $1,5 \pm 1,7$ & $1,7 \pm 1,5$ & $0,7 \pm 1,0$ \\
\hline$a_{2}$ & $6,9 \pm 0,9$ & $6,3 \pm 1,0$ & $6,2 \pm 0,5$ \\
\hline$t_{0}$ & $33,7 \pm 19,1$ & $31,0 \pm 18,6$ & $46,0 \pm 13,0$ \\
\hline$t_{0,5}$ & $41,7 \pm 15,6$ & $46,5 \pm 6,6$ & $58,5 \pm 20,4$ \\
\hline$d_{\dagger}$ & $4,1 \pm 5,6$ & $7,8 \pm 8,9$ & $5,4 \pm 3,7$ \\
\hline$t_{t}$ & $53,0 \pm 11,5^{a}$ & $63,7 \pm 13,3$ & $71,0 \pm 28,0^{a}$ \\
\hline
\end{tabular}

a $\mathrm{p}<0,05$ = teste $\dagger$ de Student) somente para a comparação entre PSD e PPDM com controle glicêmico inadequado. PSD = puérpera sem diabetes; PPDM (puérpera portadora de diabetes melito; $a_{1}=$ concentração média de lactose em $\mathrm{g} / \mathrm{dl}$ na lactogênese $\mathrm{l} ; \mathrm{a}_{2}=$ concentração média de lactose em $\mathrm{g} / \mathrm{dl}$ na lactogênese Il; $t_{0}=$ tempo inicial da transição em horas; $\dagger_{0.5}=$ metade do período de transição em horas; $d_{t}=4$ vezes $d_{t}$ representa o valor da amplitude da transição em horas; e $t_{t}=$ tempo total necessário em horas para alcançar a concentração média de lactose na lactogênese Il.

para os níveis da lactogênese II. Esta observação está de acordo com o rápido aumento, descrito na literatura (10) para a concentração de lactose no colostro entre 24 e 48 horas após o parto, para alcançar um patamar em torno de $7 \mathrm{~g} / \mathrm{dl}$, típico da lactogênese II $(7,8,11)$.

$O$ parâmetro $t_{t}$ definido pela equação de Boltzmann revelou, neste estudo, um atraso de 15 horas na transição da lactogênese I para a lactogênese II entre as PPDM em relação as PSD (Tabela 5). Esta diferença está em concordância com a faixa de variação de 15 a 28 horas reportada na literatura $(10,32-34)$. Considerando somente as pacientes com controle metabólico inadequado no grupo das PPDM (Tabela 6), este período de atraso em relação a todo o grupo das PSD foi de 18 horas.

Uma vez que a idade materna, o IMC pré-gestacional, o ganho de peso durante a gestação, o número e o tipo de parto e a idade gestacional dos recém-nascidos foram controlados neste estudo, é improvável que o atraso na lactogênese II tenha sido por causa das diferenças relacionadas a essas variáveis (Tabela 1 ).

$\mathrm{O}$ maior peso de nascimento dos FPPDM (Tabela 1) está certamente associado à macrossomia, explicada por hiperinsulinismo fetal decorrente da hiperglicemia materna e fetal $(35,36)$. A maior freqüência de internação de FPPDM na unidade neonatal em relação aos FPSD deveu-se principalmente a hipoglicemia neonatal, que é o distúrbio metabólico mais comum em FPPDM (36). A hipoglicemia neonatal requer tratamento rápido e adequado, por meio de infusão endovenosa de glicose e introdução de alimentação enteral o mais precocemente possível $(36,37)$.
Alguns pesquisadores afirmam que a sucção ou a remoção do leite durante o segundo e o terceiro dia após o parto é desnecessária para promover o estágio II da lactogênese $(38,39)$, e que "a descida do leite" ocorre, mesmo sem a sucção do bebê, até o terceiro ou quarto dia após o parto (40). Segundo Ferris e cols. (41), a separação do recém-nascido de sua mãe é uma causa importante de atraso na lactação e na produção de volume inadequado de leite por PPDM. A unidade neonatal do HC-UFU mantém conduta de incentivo ao aleitamento materno com rotinas que facilitem essa prática, mesmo se os bebês vierem a ser separados de suas mães. Neste estudo, a maioria das crianças dos dois grupos sugou o seio materno desde as primeiras horas de vida, não sendo, portanto, a causa do atraso da lactogênese II. De fato, nos cinco primeiros dias após o parto, não houve diferença significativa na concentração de lactose no colostro das mulheres PSD cujos filhos foram internados na unidade neonatal em relação às mulheres PSD cujos filhos permaneceram no alojamento conjunto (Tabela 4).

A menor concentração de lactose no colostro das PPDM no terceiro e no quinto dia do puerpério (Tabela 3) sugere que o principal fator determinante da diferença é intrínseco à condição de saúde das pacientes (15). Estes achados concordam com Arthur e cols. (10), os quais constataram que a concentração máxima de lactose no leite de mulheres diabéticas ocorreu significativamente mais tarde $(72 \pm 13$ horas após o parto) em relação às mulheres não-diabéticas $(53 \pm 12$ horas após o parto $)$. Como as PPDM com controle glicêmico inadequado, diferentemente daquelas com controle glicêmico ade- 
quado, apresentaram o tempo total $\left(\mathrm{t}_{t}\right)$ para atingir a lactogênese II significantemente maior em relação às PSD (Tabela 6), é provável que o controle inadequado da glicemia tenha sido um fator determinante do atraso da lactogênese no grupo das PPDM. Esse resultado concorda com outros relatos da literatura $(33,39,42)$.

As possíveis causas para o retardo da lactogênese em pacientes portadoras de diabetes melito podem estar relacionadas à atuação da insulina na captação de glicose pela glândula mamária. A insulina é importante no controle do fluxo de nutrientes para a glândula mamária durante a lactação $(43,44)$, provavelmente por potencializar os efeitos da prolactina (45), que regula a expressão de transportadores de glicose para a glândula mamária, como demonstrado em experimentos com animais (46). Isso, entretanto, não exclui a possibilidade de que outros fatores, mais difíceis de serem quantificados, como a dificuldade no trabalho de parto e o grau de estresse da mãe ou do bebê, possam ter sido também importantes no atraso para alcançar a lactogênese II (47-49).

Uma vez que a excelência do leite humano para lactentes não constitui motivo de dúvida, o atraso de 18 horas no início da lactogênese II em mulheres portadoras de $\mathrm{DMl}$ com controle glicêmico inadequado representa um fato de grande relevância. Sugere a necessidade de se prestar melhor atendimento a estas pacientes pelos profissionais da área da saúde, com implementação de orientações no pré-natal por equipe multiprofissional de saúde, para incentivar e apoiar o aleitamento materno. $\mathrm{O}$ pediatra deve dar atenção especial a esses recém-nascidos filhos de mulheres portadoras de diabetes, no sentido de garantir uma oferta nutricional adequada, uma vez que estes bebês apresentam risco aumentado para desenvolver hipoglicemia neonatal.

Este estudo mostrou que puérperas portadoras de DMl apresentaram atraso significante na transição da fase I para a fase II da lactogênese, provavelmente por causa do controle glicêmico inadequado, dificultando o estabelecimento do aleitamento materno. É importante manter um bom controle glicêmico das pacientes portadoras de $\mathrm{DMl}$ após o parto, monitorando os fatores que alterariam os níveis de glicose sanguínea, como a dieta materna e a insulinoterapia.

\section{REFERÊNCIAS}

1. Cregan MD, Hartmann PE. Computerized breast measurement from conception to weaning: clinical implications. J Human Lact. 1999;15:89-96.
2. Arthur PG, et al. Lactose in blood in nonpregnant, pregnant and lactating women. J Pediatr Gastroenterol Nutr. 1991;13:254-9.

3. Butte NF, Garza C, Smith EO. Variability of macronutrient concentrations in human milk. Eur J Clin Nutr. 1988;42:345-9.

4. Lamounier JA, Vieira GO, Gouvêa LC. Composição do leite humano: fatores nutricionais. In: Rego JD, editor. Aleitamento materno. São Paulo:Atheneu; 2002. p. 47-58.

5. Lawrence RA, Lawrence RM. Breastfeeding: a guide for the medical profession. $6^{\text {th }}$ ed. Saint Louis:Mosby, 2005.

6. Buhimschi CS. Endocrinology of lactation. Obstet Gynecol Clin North Am. 2004;31:963-79.

7. Calil VMT, Falcão MC. Composição do leite humano. In: Feferbaum R, Falcão MC, editores. Nutrição do recém-nascido. São Paulo: Atheneu; 2003. p. 215-27.

8. Garza C, Hopkinson JM. Physiology of lactation. In: Tsang RC, et al. Nutrition during infancy. Philadelphia: Hanley \& Belfus; 1988. p. 20-32.

9. Lawrence R. Successful breastfeeding. Am J Dis Child. 1981;135:595-6.

10. Arthur PG, Smith M, Hartmann PE. Milk lactose, citrate, and glucose as markers of lactogenesis in normal and diabetic women. J Pediatr Gastroenterol Nutr. 1989;9(4):488-96.

11. Saint $L$, Smith M, Hartmann PE. The yield and nutrient content of colostrums and milk of women from birth to 1 month postpartum. Br J Nutr. 1984;52:87-95.

12. Giugliani ERJ, Victora CG. Normas alimentares para crianças brasileiras menores de dois anos: bases científicas. Brasília: Organização Pan-Americana de Saúde, Organização Mundial de Saúde; 1997.

13. Victora CG, Smith PG, Vaughan JP, Lombard C, Teixeira AMB. Evidence for protection by breast-feeding deaths from infectious diseases in Brazil. Lancet. 1987;2:319-22.

14. Freund C, Mirabel L, Annane K, Mathelin C. Breastfeeding and breast cancer. Gynecol Obstet Fertil. 2005;33:739-44.

15. Neubauer SH. Lactation in insulin-dependent diabetes. Prog Food Nutr Sci. 1990;14(4):333-70.

16. Brasil. Ministério da Saúde. Gestação de alto risco: manual técnico. $3^{\mathrm{a}}$ ed. Brasília:Ministério da Saúde; 2000.

17. Schmidt MI, Reichelt AJ. Consenso sobre diabetes gestacional e pré-gestacional. Arq Bras Endocrinol Metab. 1999;43:14-8.

18. Ryan EA. Pregnancy in diabetes. Med Clin North Am. 1998;82:823-45.

19. Gerstein HC. Cow's milk exposure and type 1 diabetes mellitus. Diabetes Care. 1994;17:13-9.

20. Malcova H, Sumnik Z, Drevinek P, Venhacova J, Lebl J, Cinek $O$. Absence of breastfeeding is associated with the risk of type 1 diabetes: a case-control study in a population with rapidly increasing incidence. Eur J Pediatr. 2006;165:114-9.

21. Stuebe AM, Rich-Edwards JW, Willett WC, Manson JE, Michels KB. Duration of lactation and incidence of type 2 diabetes. JAMA. 2005;294:2601-10.

22. World Health Organization. 54 $4^{\text {th }}$ World Health Assembly, Geneva, 2001.

23. American Dietetic Association. Position of the American Dietetic Association: breaking the barriers to breastfeeding. $J$ Am Diet Assoc. 2001;101:1213-20.

24. Sociedade Brasileira de Diabetes. Consenso brasileiro sobre diabetes 2002: diagnóstico e classificação do diabetes mellitus e tratamento do diabetes mellitus tipo 2. Rio de Janeiro: Diagraphic, 2003. 
25. American Diabetes Association. Diagnosis and classification of diabetes mellitus. Diabetes Care. 2005;28 (Suppl) 1:37-42.

26. American Diabetes Association. Standards of medical care in diabetes. Diabetes Care. 2007;30:4-11.

27. Costa THM, Dórea JG. Concentration of fat, protein, lactose and energy in milk of mothers using hormonal contraceptives. Ann Trop Paediatr. 1992;12:203-9.

28. Penha-Silva N, Fonseca AM, Brito AG, Souza-Penha MA, Ferreira TAA. Rapid determination of reducing sugars with picric acid for biotechnological use. Biosci J. 2004;20(3):183-8.

29. Sacks DB. Glicídios. In: Burtis CA, Ashwood ER, editores. Tietz fundamentos de química clínica. $4^{\mathrm{a}}$ ed. Rio de Janeiro: Guanabara Koogan; 1998. p. 341-63.

30. Bussab WO, Morettin P. Estatística básica. $5^{\mathrm{a}}$ ed. São Paulo: Saraiva; 2002.

31. Siegel S, Castellan NJ. Nonparametric statistics for the behavioral sciences. New York: McGraw Hill, 1988.

32. Hartmann PE, Cregan MD. Lactogenesis and the effects of insulin-dependent diabetes mellitus and prematurity. J Nutr. 2001;131 Suppl 1:3016-20.

33. Neubauer SH, Ferris AM, Chase CG, Fanelli J, Thompson CA, Lammi-Keefe $\mathrm{CJ}$, et al. Delayed lactogenesis in women with insulin-dependent diabetes mellitus. Am J Clin Nutr. 1993;58(1):54-60.

34. Neville MC, Allen JC, Archer PC, Casey CE, Seacat J, Keller RP, et al. Studies in human lactation: milk volume and nutrient composition during weaning and lactogenesis. Am J Clin Nutr. 1991;54(1):81-92.

35. Melo G, Parretti E, Mecacci F, Torre PL, Cioni R, Cianciulli D, et al. What degree of maternal metabolic control in women with type 1 diabetes is associated with normal body size and proportions in full-term infants? Diabetes Care. 2000;23:1494-8.

36. Nold JL, Georgieff MK. Infants of diabetic mothers. Pediatr Clin North Am. 2004;51:619-37.

37. Barros MCM. Assistência ao recém-nascido de mãe diabética. In: Kolpelman Bl, et al. Diagnóstico e tratamento em neonatologia. São Paulo: Atheneu; 2004. p. 25-30.

38. Kulski JK, Hartmann PE. Changes in human milk composition during the initiation of lactation. Austr J Exp Biol Med Sci. 1981;59(1):101-14.
39. Neville MC, Morton J, Umemura S. Lactogenesis, the transition from pregnancy to lactation. Pediatric Clin North Am. 2001;48:35-52.

40. Giugliani ERJ. Problemas comuns na lactação e seu manejo. J Ped. 2004;80 (Suppl) 5:147-54.

41. Ferris AM, Neubauer SH, Bendel RB, Green KW, Ingardia CJ, Reece EA. Perinatal lactation protocol and outcome in mothers with and without insulin-dependent diabetes mellitus. Am J Clin Nutr. 1993;58(1):43-8.

42. Ostrom KM, Ferris AM. Prolactin concentrations in serum and milk of mothers with and without insulin-dependent diabetes mellitus. Am J Clin Nutr. 1993;58:49-53.

43. Neville MC, Sawicki V, Hay Jr WW. Effect of fasting, elevated plasma glucose and plasma insulin concentrations on milk secretion in women. J Endocrinol. 1993;139:165-73.

44. Neville MC, McFadden TB, Forsyth I. Hormonal regulation of mammary differentiation and milk secretion. J Mammary Gland Biol Neoplasia. 2002;7:49-66.

45. Duclos M, Houdebine LM, Djiane J. Comparison of insulin-like growth factor1 and insulin effects on prolaction-induced lactogenesis in rabbit mammary gland in vitro. Mol Cell Endocrinol. 1989;65:129-34.

46. Zhao FQ, Miller Pj, Wall EH, Zheng YC, Dong B, Neville MC, et al. Bovine glucose transporter GLUT8: cloning, expression, and developmental regulation in mammary gland. Biochim Biophys Acta. 2004;1680:103-13.

47. Chapman DJ, Perez-Escamilla R. Identification of risk factors for delayed onset of lactation. J Am Diet Assoc. 1999;99:450-4.

48. Chen CD, Nommsen-Rivers L, Dewey KG, Lönnerdal B. Stress during labor and delivery and early lactation performance. Am J Clin Nutr. 1998;68:335-44.

49. Dewey KG. Maternal and fetal stress is associated with impaired lactogenesis in humans. J Nutr. 2001;131 (Suppl) 1:3012-5.

Endereço para correspondência:

Paulo Tannus Jorge

Rua Cruzeiro dos Peixotos, 499, sala 303 - Centro

38400-608, Uberlândia MG

E-mail: pauloj@triang.com.br 